\title{
A Note On The Defininiton of An Orlicz Space
}

\author{
Alen Osançlıol \\ Yeditepe Üniversitesi, Fen ve Edebiyat Fakültesi, Matematik Bölümü, 34755 Kayışdağı-Istanbul. \\ e-mail: osancliola@yahoo.com
}

Geliş Tarihi:09.07.2014 ; Kabul Tarihi:05.12.2014

\begin{tabular}{cl} 
& Abstract \\
\cline { 2 - 3 } Key words & The Orlicz spaces were introduced by Z.W. Birnbaum and W. Orlicz in 1931 as a natural generalization \\
Orlicz space; & of the classical Lebesgue spaces. For this generalization the function $x^{p}$ entering in the definition of \\
N-Function; & Lebesgue's space is replaced by a more general convex function $\Phi$. Until today, Orlicz spaces have been \\
Young function; & studied by many authors from a different aspects. But, most of these studies used different definitions \\
Orlicz function; & $\begin{array}{l}\text { for the convex function } \Phi \text { to define the Orlicz spaces. In this paper we will investigate the exact } \\
\text { Lebesgue space }\end{array}$ \\
$\begin{array}{l}\text { relationships between these different definitions of convex functions } \Phi \text { and obtain the differences } \\
\text { between the corresponding Orlicz spaces. }\end{array}$
\end{tabular}

\section{Orlicz Uzayının Tanımı Üzerine Bir Not}

\author{
Anahtar kelimeler \\ Orlicz uzayı; \\ N-Fonksiyonu; \\ Young fonksiyonu; \\ Orlicz fonksiyonu; \\ Lebesgue uzayı
}

Özet

Orlicz uzayları 1931 yılında Z.W. Birnbaum ve W. Orlicz tarafından klasik Lebesgue uzaylarının doğal bir genelleştirmesi olarak tanıtılmıştır. Bu genelleştirme Lebesgue uzayları tanımında yer alan $x^{p}$ fonksiyonu yerine daha genel bir $\Phi$ konveks fonksiyonu alınarak yapılmıştır. Günümüze kadar Orlicz uzayları pek çok yazar tarafından farklı bakış açılarıyla çalışılmıştır. Fakat bu çalışmaların çoğunda Orlicz uzayı tanımındaki konveks $\Phi$ fonksiyonu için farklı tanımlar kullanılmıştır. Bu çalışmada, Orlicz uzayları tanımında adı geçen $\Phi$ konveks fonksiyonlarının farklı tanımları arasındaki ilişkiler net bir şekilde incelenecek ve bu farklı fonksiyonlara karşı gelen Orlicz uzayları arasındaki farklııklar belirlenecektir.

(c) Afyon Kocatepe Üniversitesi

\section{Introduction}

The Orlicz spaces were introduced by Z.W. Birnbaum and W.Orlicz (1931) as a natural generalization of the classical Lebesgue spaces $L^{p}$, $1<p<+\infty$. For this generalization the function $x^{p}$ entering in the definition of $L^{p}$ space is replaced by a more general convex function $\Phi$, which is called an $\mathrm{N}$-function and he studied on the Orlicz space associated to $\mathrm{N}$-function $\Phi$. The first detailed study on Orlicz spaces was given by Krasnosel'skii and Rutickii (1961) and they considered the function $\Phi$ as an $\mathrm{N}$-function.
The following definition of an N-function is based on the integral representation of the real valued convex function.

Definition 1.1: (Krasnosel'skii and Rutickii (1961)) Let $\varphi:[0,+\infty) \rightarrow[0,+\infty)$ be a right continuous function for $t \geq 0$ and $\varphi(t)$ is increasing for $t>0$ and satisfy the conditions $\varphi(0)=0$ and $\lim _{t \rightarrow \infty} \varphi(t)=+\infty$. Then the function $\Phi:[0,+\infty) \rightarrow[0,+\infty)$ defined as

$$
\Phi(x)=\int_{0}^{x} \varphi(t) d t, \mathrm{x} \geq 0
$$


is called an $\mathrm{N}$-function.

On the other hand, equivalent definition of $\mathrm{N}$ function is given as follows and we would prefer to use the following definition for the $\mathrm{N}$-function.

Definition 1.2: (Krasnosel'skii and Rutickii (1961)) (N-Function) A continuous convex function $\Phi:[0,+\infty) \rightarrow[0,+\infty)$ which satisfy the conditions

$$
\lim _{x \rightarrow 0^{+}} \Phi(x)=0 \text { and } \lim _{x \rightarrow+\infty} \Phi(x)=+\infty
$$

is called an $\mathrm{N}$-function.

Example 1.3: The following functions are $\mathrm{N}$ functions.

1) $\Phi(\mathrm{x})=\frac{x^{p}}{p}, x \geq 0$ with $p>1$

2) $\Phi(\mathrm{x})=\mathrm{e}^{\mathrm{x}}-\mathrm{x}-1, x \geq 0$

3) $\Phi(\mathrm{x})=\mathrm{x}^{\mathrm{p}} \cdot \log (1+x)^{\alpha}$ with $p \geq 1$ and $\alpha>0$.

Nonetheless, in some works this $\mathrm{N}$-function $\Phi$ is called as a Young function Kufner et al. (1977).

On the other hand, the more extensive studies, which also includes the applications of Orlicz spaces, are given in Rao and Ren (1991, 2002). In these studies the function $\Phi$ is taken as a Young function differently from $\mathrm{N}$-function.

Definition 1.4: (Rao and Ren (2002)) (Young Function) A convex function $\Phi:[0,+\infty) \rightarrow$ $[0,+\infty]$ which satisfy the conditions

$$
\Phi(0)=\lim _{x \rightarrow 0^{+}} \Phi(x)=0
$$

and

$$
\lim _{x \rightarrow+\infty} \Phi(x)=+\infty
$$

is called a Young function.

Example 1.5: The following functions are Young functions.
1) $\Phi(\mathrm{x})=\frac{x^{p}}{p}, x \geq 0$ with $p \geq 1$

2) Let $0<a<b<+\infty$,

$$
\Phi(x)=\left\{\begin{array}{c}
(x-a)^{p}, a \leq x<b \\
+\infty, x \geq b
\end{array}\right.
$$

Remark 1.6: Note that, $\mathrm{N}$-function and Young function are defined on $\mathbb{R}$ and $\Phi$ is taken an even function in Krasnosel'skii and Rutuckii (1961), Rao and Ren (1991) respectively. But in this paper we take the domain of the $\Phi$ as $[0,+\infty)$ for the convenience with the other definitions.

Also, we remark that an $\mathrm{N}$-function $\Phi$ is finite real valued convex function defined on $[0,+\infty)$, so this implies that $\Phi$ is necessarily continuous. However, a Young function can have infinite value at a point, and hence may be discontinuous at such a point.

Moreover, recently, in several studies about Orlicz spaces especially on the composition operators (Arora and et al. (2007), Kumar (1997), Raj and Khosla (2009)), the function $\Phi$ is defined differently from the Young function used in Rao and Ren's works but again they called this new function $\Phi$ as a Young function. Since, this new function $\Phi$ has stronger conditions than the previous one, through this paper this function $\Phi$ is called a strong Young function instead of a Young function.

Definition 1.7: (Strong Young Function) A convex continuous function $\Phi:[0,+\infty) \rightarrow[0,+\infty)$ which satisfy the conditions $\Phi(x)=0 \Leftrightarrow x=0$ and

$$
\lim _{x \rightarrow+\infty} \Phi(x)=+\infty
$$

is called a strong Young function.

Example 1.8: The following functions are strong Young functions.

1) $\Phi(x)=e^{x^{\beta}}-1, x \geq 0$ with $\beta>0$.

2) $\Phi(x)=\cosh x-1, x \geq 0$. 
Again, in some papers (for instance in Hudzik et al. (1987)) the function $\Phi$ is defined differently from the other definitions of function $\Phi$ and called Orlicz function.

Definition 1.9: (Orlicz Function) Let $\Phi:[0,+\infty) \rightarrow$ $[0,+\infty]$ be a convex function such that $\Phi(0)=0$, $\lim _{x \rightarrow+\infty} \Phi(x)=+\infty$ and which is not identically zero or infinity on $(0,+\infty)$ (i.e. $\exists x, y \in(0,+\infty)$, $\Phi(x) \neq 0$ and $\Phi(y) \neq+\infty)$.

If $\Phi$ is left continuous at $b_{\Phi}$, where

$$
b_{\Phi}=\sup \{x>0 \mid \Phi(x)<+\infty\}
$$

then $\Phi$ is called an Orlicz function. Similarly another important constant connected with the Orlicz function $\Phi$ is $a_{\Phi}=\inf \{x>0 \mid \Phi(x)>0\}$. Consequently, the Orlicz function $\Phi$ is increasing and $0 \leq a_{\Phi} \leq b_{\Phi} \leq+\infty$.

Moreover, the following proposition can be given for the Orlicz function.

Proposition 1.10: Let $\Phi:[0,+\infty) \rightarrow[0,+\infty]$ be an Orlicz function. Then

(i) $\quad a_{\Phi}=0 \Leftrightarrow(\Phi(x)=0 \Leftrightarrow x=0)$.

(ii) $b_{\Phi}=+\infty \Leftrightarrow \forall x>0, \Phi(x)<+\infty$.

Proof: Proof of (i) :

$\Rightarrow$ : Assume $a_{\Phi}=\inf \{x>0 \mid \Phi(x)>0\}=0$ for the Orlicz function $\Phi$. If $x=0$ then $\Phi(x)=0$ is clear. Conversly if $\Phi(x)=0$ we will show that $x=0$. Suppose that $x>0$. Defining $A=$ $\{x>0 \mid \Phi(x)>0\}$, we obtain

$$
\forall \varepsilon>0, \exists x^{\prime} \in A, x^{\prime}<\varepsilon .
$$

Here, if we take $\varepsilon=x>0$, then there exists $\mathrm{x}^{\prime} \in A$ such that $x^{\prime}<\varepsilon=x$. Consequently $\Phi\left(x^{\prime}\right)>0$. On the other hand we can find an $\alpha \in[0,1]$ such that $x^{\prime}=\alpha x$, since $0<x^{\prime}<x$. Moreover, we obtain that $0 \leq \Phi\left(x^{\prime}\right)=\Phi(\alpha x) \leq \alpha \Phi(x)=0$ because $\Phi$ is convex. So $\Phi\left(x^{\prime}\right)=0$ but this contradicts with $\Phi\left(x^{\prime}\right)>0$.

$\Leftarrow$ : Suppose that $\Phi(x)=0$ if and only if $x=0$. So, we have $\Phi(x)>0 \Leftrightarrow x>0$ from the definition of Orlicz function. And we have $\Phi\left(\frac{1}{n}\right)>0$ for all $n \in \mathbb{N}$. If we take $A=\{x>0 \mid \Phi(x)>0\}$ then $\frac{1}{n} \in \mathbb{N}$ for all $n \in \mathbb{N}$. From here we get $\inf A \leq \frac{1}{n}$ and so we obtain $a_{\Phi}=\inf A=0$.

Proof of (ii):

$\Rightarrow$ : Assume that $b_{\Phi}=+\infty$. If we define $B=\{x>0 \mid \Phi(x)<+\infty\}$ then $B$ is not a bounded set. So we get

$$
\forall x>0, \exists x^{\prime} \in B, x^{\prime}>x .
$$

For this $x^{\prime}$ in $\mathrm{B}$, we know that $\Phi\left(x^{\prime}\right)<+\infty$. So we can find an $\alpha \in[0,1]$ such that $x=\alpha x^{\prime}$. Moreover, we obtain

$$
\Phi(x)=\Phi\left(\alpha x^{\prime}\right) \leq \alpha \Phi\left(x^{\prime}\right)<+\infty
$$

by using conveity of $\Phi$. Thus the proof of the first part is completed.

$\Leftarrow$ : Conversely, suppose that $\Phi(x)$ is finite for all $x>0$. Then $(0,+\infty) \subseteq B$ where $B=\{x>0 \mid \Phi(x)<+\infty\}$. So it is clear that $b_{\Phi}=\sup B=+\infty$.

In this paper, we will give the different definitions of convex functions $\Phi$ which are used to define the Orlicz spaces and investigate the relationships between these different definitions. Also, we will consider the corresponding Orlicz spaces to the convex functions $\Phi$ mentioned above and for all cases we determine that which Lebesgue spaces $L^{p}$ can be obtained from an Orlicz spaces.

\section{Main Result}

In this part, we will investigate the relationship between the $\mathrm{N}$-function, Young function, strong Young function and Orlicz function.

\subsection{Relationships Between Convex Functions $\Phi$}

Theorem 2.1: If $\Phi$ is an $\mathrm{N}$-function then $\Phi$ is a strong Young function.

Proof: Assume that $\Phi$ is an $\mathrm{N}$-function. Then $\Phi:[0,+\infty) \rightarrow[0,+\infty)$ is a convex continuous function and satisfy the conditions

$\lim _{x \rightarrow 0^{+}} \frac{\Phi(x)}{x}=0 \quad$ and $\quad \lim _{x \rightarrow+\infty} \frac{\Phi(x)}{x}=+\infty$. 
Firstly, from the definition of $\lim _{x \rightarrow+\infty} \frac{\Phi(x)}{x}=+\infty$, we get

$$
\forall M>0, \exists x_{M}>0, x>x_{M} \Rightarrow \frac{\Phi(x)}{x}>M .
$$

Here $x_{M}$ can be taken as $x_{M}>1$. Then for all $x>x_{M}>1$ we write

$$
\Phi(x)>M x>M x_{M}>M .
$$

Thus $\lim _{x \rightarrow+\infty} \Phi(x)=+\infty$. On the other hand, from the definition of $\lim _{x \rightarrow 0^{+}} \frac{\Phi(x)}{x}=0 \quad$ we get

$$
\forall \varepsilon>0, \exists \delta_{\varepsilon}>0,0<x<\delta_{\varepsilon} \Rightarrow \frac{\Phi(x)}{x}<\varepsilon .
$$

Here $\delta_{\varepsilon}$ can be taken as $\delta_{\varepsilon}<1$. So we have

$$
\Phi(x)<\frac{\Phi(x)}{\delta_{\varepsilon}}<\frac{\Phi(x)}{x}<\varepsilon
$$

for all $0<x<\delta_{\varepsilon}<1$. This says that $\lim _{x \rightarrow 0^{+}} \Phi(x)=0$. Then $\Phi(0)=\lim _{x \rightarrow 0^{+}} \Phi(x)=0$, since $\mathrm{N}$-function $\Phi$ is continuous at $x=0$.

Moreover, $\Phi(x)>0$ when $x>0$, since every $\mathrm{N}$ function is strictly increasing. In other words we have $\Phi(x)=0$ if and only if $x=0$. Consequently, $\Phi$ is a strong Young function.

Theorem 2.2: If $\Phi$ is a strong Young function then $\Phi$ is an Orlicz function.

Proof: Let $\Phi$ be a strong Young function. Then, $\Phi:[0,+\infty) \rightarrow[0,+\infty)$ is a convex continuous function which satisfy the conditions $\lim _{x \rightarrow+\infty} \Phi(x)=+\infty$ and $\Phi(x)=0 \Leftrightarrow x=0$.

From here, it is clear that $\Phi$ in not identically zero or infinity. Also, we see that $a_{\Phi}=0$ and $b_{\Phi}=+\infty$ by using Proposition 1.10. In addition $\Phi$ is left continuous at $b_{\Phi}=+\infty$ since $\lim _{x \rightarrow+\infty} \Phi(x)=$ $+\infty$. So, $\Phi$ is an Orlicz function.

Theorem 2.3: If $\Phi$ is an Orlicz function then $\Phi$ is a Young function.

Proof: Assume that $\Phi$ is an Orlicz function. From the definition of an Orlicz function we know that $\Phi$ is convex and satisfy the conditions $\Phi(x)=0$ and $\lim _{x \rightarrow+\infty} \Phi(x)=+\infty$. So, we have to show that an Orlicz function $\Phi$ is right continuous at $x=0$ to obtain $\Phi$ is a Young function. In other words we have to show that

$$
\forall \varepsilon>0, \exists \delta_{\varepsilon}>0,0<x<\delta_{\varepsilon} \Rightarrow 0 \leq \Phi(x)<\varepsilon .
$$

For this purpose, take an arbitrary $\varepsilon>0$. If we consider the number $a_{\Phi}=\inf \{x>0 \mid \Phi(x)>0\}$ then there exists two cases for $a_{\Phi}$.

Firstly, assume that $a_{\Phi}>0$. Then, $\Phi(x)=0$ for all $x \in\left(0, a_{\Phi}\right)$ from the definition of infimum. If we take $\delta_{\varepsilon}=a_{\Phi}>0$ then $\Phi(x)=0<\varepsilon$ for all $0<x<\delta_{\varepsilon}$. Thus $\Phi$ is right continuous at $x=0$.

Secondly, if $a_{\Phi}=0$ then we get $\Phi(x)>0$ for all $x>0$ from Proposition 1.10. Since an Orlicz function is not identical infinity on $(0,+\infty)$, there exists $x_{0} \in(0,+\infty)$ such that $0<\Phi\left(x_{0}\right)<+\infty$.

We obtain from here that for all $\varepsilon>0$ there exists $x_{\varepsilon} \in(0,+\infty)$ such that $0<\Phi\left(x_{\varepsilon}\right)<+\infty$. Indeed, if $\Phi\left(x_{0}\right)<\varepsilon$ then $\Phi\left(x_{\varepsilon}\right)<+\infty$ for $x_{\varepsilon}=x_{0}$. If $\Phi\left(x_{0}\right) \geq \varepsilon$ then for the number $x_{\varepsilon}=\alpha x_{0}$ where $0 \leq \alpha=\frac{\varepsilon}{2 \Phi\left(x_{0}\right)} \leq 1$, we have

$$
\Phi\left(x_{\varepsilon}\right)=\Phi\left(\alpha x_{0}\right) \leq \alpha \Phi\left(x_{0}\right) \leq \frac{\varepsilon}{2}<\varepsilon
$$

Since $\Phi$ is convex. Therefore, if $\delta_{\varepsilon}=x_{\varepsilon}>0$ we obtain

$$
0 \leq \Phi(x) \leq \Phi\left(\delta_{\varepsilon}\right)=\Phi\left(x_{\varepsilon}\right)<\varepsilon
$$

for all $0<x<\delta_{\varepsilon}$ since an Orlicz function $\Phi$ is increasing.

On the other hand, the converse of all the above theorems are not true. To see this we can give the following counterexamples.

A strong Young function $\Phi$ may not be an $N$ function.

Example 2.4: If $\Phi(x)=e^{x}-1$ for all $x \geq 0$ then it is clear that $\Phi$ is a strong Young function. But this function $\Phi$ is not an $\mathrm{N}$-function, since

$$
\lim _{x \rightarrow 0^{+}} \frac{\Phi(x)}{x}=\lim _{x \rightarrow 0^{+}} \frac{e^{x}-1}{x}=1 \neq 0 .
$$


An Orlicz function $\Phi$ may not be a strong Young function.

Example 2.5: Let $a>0$ be a constant then the function defined by

$$
\Phi(x)=\left\{\begin{array}{c}
0,0 \leq x<a \\
x-a, x \geq a
\end{array}\right.
$$

is an Orlicz function. Even though this function $\Phi$ is continuous, it has value zero at a non-zero point $x$ $(\Phi(a)=0)$. So, $\Phi$ is not a strong Young function.

A Young function $\Phi$ may not be an Orlicz function.

Example 2.6: Let

$$
\Phi(x)=\left\{\begin{array}{l}
x, 0 \leq x<1 \\
+\infty, x \geq 1
\end{array}\right.
$$

It is easy to see that $\Phi$ is a Young function. Moreover, $\Phi$ is not identical zero or infinity. But, we have

$$
b_{\Phi}=\sup \{x>0 \mid \Phi(x)<+\infty\}=1
$$

and $\Phi$ is not left continuous at $b_{\Phi}=1$, since

$$
\lim _{x \rightarrow b_{\Phi}} \Phi(x)=1 \neq+\infty=\Phi(1) .
$$

So, $\Phi$ is not an Orlicz function.

Therefore we get the following corollaries for the functions $\Phi$.

Corollary 2.7: For the function $\Phi$, we have

$\mathrm{N}-$ function $\Rightarrow$ Strong Young function $\Rightarrow$ Orlicz function $\Rightarrow$ Young function.

Corollary 2.8: For the function $\Phi$, we have

$\mathrm{N}-$ function $\nLeftarrow$ Strong Young function $\nLeftarrow$ Orlicz function $\nLeftarrow$ Young function

\section{Discussion and Result}

In this study we investigate the relationships between the different defininitons of convex functions $\Phi$ which are used to define Orlicz space. Now we will give the definition of the Orlicz space determined by convex function $\Phi$ and we obtain the differences between the corresponding Orlicz spaces.

Let $(X, \Sigma, \mu)$ be a $\sigma$ - finite measure space and $\Phi$ be a Young function. We denote by $L^{\Phi}(X)$, the Orlicz space associated to function $\Phi$ and defined by

$$
L^{\Phi}(X):=\left\{f: X \rightarrow \mathbb{R} \mid \int_{X} \Phi(\alpha|f|) d \mu<+\infty, \text { some } \alpha>0\right\}
$$

where $f$ indicates the $\mu$-equivalence classes of measurable functions.

Example 3.1: If $\Phi(x)=\frac{x^{p}}{p}$ where $1 \leq p<+\infty$, then the corresponding Orlicz space $L^{\Phi}(X)$ is equal to the usual Lebesgue space $L^{p}(X)$.

Example 3.2: In case of $p=+\infty$, consider the Young function $\Phi$ defined by

$$
\Phi(x)=\left\{\begin{array}{l}
0,0 \leq x \leq 1 \\
+\infty, x>1
\end{array}\right.
$$

then the corresponding Orlicz space $L^{\Phi}(X)$ is equal to the essentially bounded functions space $L^{\infty}(X)$.

Now we will consider different definitions of convex functions $\Phi$ and examine that which $L^{p}(X)$ spaces can be obtained from an Orlicz spaces $L^{\Phi}(X)$ in all different cases.

If $\Phi$ is an $\mathrm{N}$-function then for $1<p<+\infty$ the $L^{p}$ spaces are obtained. But, Banach function spaces, even Banach algebras $L^{1}$ and $L^{\infty}$ spaces can not be obtained as a special case from an Orlicz spaces. Consequently the definition of $\mathrm{N}$-function is considerably restrictive.

If $\Phi$ is considered as a strong Young function, then the Lebesgue spaces $L^{p}$ where $1 \leq p<+\infty$ are obtained. However, for the limit case $p=+\infty$ the essentially bounded measurable functions space $L^{\infty}$ can not be obtained from an Orlicz space.

On the other hand, if we take $\Phi$ as a Young function or Orlicz function then all $L^{p}$ spaces for $1 \leq p \leq+\infty$ are obtained.

So, for studying Orlicz spaces it is convenient to use Young function definition in general to define the Orlicz space. 
Remark 3.3: We note that if we consider the functions $\Phi$ given in Example 1.3, 3) and Example $1.8,1)$ then we obtain the another useful examples of Orlicz spaces so called Zygmund spaces denoted by $L^{p}(\log L)^{\alpha}(\mathrm{X})$ and $\operatorname{Exp} L^{\beta}(\mathrm{X})$ respectively (Bennet and Sharpley 1988).

\section{Acknowledgement}

I would like to thank S. Öztop for her helpful suggestions. Also I would like to mention that this research is supported by Scientific Research Projects Coordination Unit of Istanbul University with project number 14671.

\section{References}

Arora, S.C., Datt, G., and Verma, S., 2007. Multiplication and Composition Operators on Orlicz-Lorentz Spaces. Int. Journal of Math. Analysis, Vol. 1, no. 25, 1227-1234.

Bennet, G. and Sharpley, R., 1988. Interpolation of Operators, Academic Press, London.

Cui, Y., Hudzik, H., Kumar, R. and Maligranda, L., 2004. Composition operators in Orlicz spaces. Journal of the Australian Mathematical Society, 76 (2), 189-206.

Hudzik, H., Kamińska, A. and Musielak, J., 1987. On Some Banach Algebras Given by a Modular. A. Haar Mem. Conf., Budapest/Hung. 1985, Colloq. Math. Soc. János Bolyai, 49, 445--463.

Krasnosel'skii, M. A., and Rutickii, Ja. B., 1961. Convex Functions and Orlicz Spaces, Noordhoff, Ltd.; 1st edition.

Kumar, R., 1997. Composition operators on Orlicz spaces. Integral Equations and operators theory, Vol. 29, 17-22.

Kufner, A., John, O. and Fucik, S., 1977. Function Spaces, Springer; 1 edition.

Birnbaum, Z. W. and Orlicz, W., 1931. Über die Verallgemeinerung des Begriffes der zueinander Konjugierten Potenzen. Studia Mathematica, 3: 1-67.

Raj, K. and Khosla, V., 2009. Weighted Composition Operators between Spaces of Orlicz-Functions. International Journal of Algebra, Vol. 3, no. 7, 315 - 324.
Rao, M. M., and Ren, Z. D., 1991. Theory of Orlicz Spaces, CRC Press; 1st edition.

Rao, M.M., and Ren., Z.D., 2002. Applications Of Orlicz Spaces, CRC Pres;1 edition. 